\title{
THE STRONG MAXIMUM PRINCIPLE FOR THE HEAT EQUATION*
}

\author{
by DAVID COLTON
}

(Received 23rd February 1984)

The strong maximum principle for harmonic functions is usually arrived at by appealing to the mean value theorem (c.f. [2], p. 53). It is also of course possible simply to appeal to the Hopf maximum principle [2], but using sledge hammers to kill flies is generally viewed as aesthetically unpleasing. In contrast to the case of harmonic functions, the only proof of the strong maximum principle for the heat equation that is known to me is to invoke Nirenberg's strong maximum principle for parabolic equations [2]. As in the case of harmonic functions, it seems desirable to provide a direct proof of this result without having to go through the subtle comparison arguments that are employed in the more general case. The purpose of this note is to provide a proof of the strong maximum principle for the heat equation based on a mean value theorem for solutions of the heat equation which we derive below. Such an approach provides a straightforward and simple proof of the strong maximum principle which avoids most of the detailed estimates of the proof of the maximum principle for more general parabolic equations. Unfortunately the proof of the maximum principle for the heat equation using the mean value theorem is not as short as the proof in the corresponding case of harmonic functions. It nevertheless seems worthwhile to show that such an alternate proof is possible, and it is to this purpose that we address this paper.

For the sake of simplicity we shall only consider the case of the heat equation in three space dimensions.

Theorem. Let $u(\mathbf{x}, t), \mathbf{x} \in \mathbb{R}^{3}$, satisfy the heat equation

$$
\Delta_{3} u=u_{t}
$$

in a domain $E$ and suppose that the maximum of $u$ in $E$ is $M$ and that it is attained at some interior point $\left(\mathbf{x}_{0}, t_{0}\right)$. If $\left(\mathbf{x}_{1}, t_{1}\right)$ is a point of $E$ which can be connected to $\left(\mathbf{x}_{0}, t_{0}\right)$ by a path in $E$ consisting only of horizontal and upward vertical line segments, then $u=M$ at $\left(\mathbf{x}_{1}, t_{1}\right)$.

Proof. Consider the spherical mean of $u$ about the point $\left(\mathbf{x}_{0}, t\right)$ defined by

$$
S(R, t)=\frac{1}{4 \pi R^{2}} \int_{\left|\mathbf{x}-\mathbf{x}_{0}\right|=R} u(\mathbf{x}, t) d s .
$$

*This research was supported in part by AFOSR Grant 81-0103. 
Then (c.f. [1], pp. 103-105) $R S(R, t)$ is a strong solution of the heat equation in one space dimension and since $R S(R, t)$ is odd we can conclude that $S(R, t)$ is an analytic function of $R$ in some neighbourhood of the origin (c.f. [1] p. 179), i.e.

$$
\begin{aligned}
S(R, t) & =\sum_{n=0}^{\infty} \frac{R^{2 n}}{(2 n+1) !} \frac{\partial^{2 n+1}}{\partial R^{2 n+1}}(R S(R, t))_{R=0} \\
& =\sum_{n=0}^{\infty} \frac{R^{2 n}}{(2 n+1) !} \frac{\partial^{n} S(0, t)}{\partial t^{n}} \\
& =\sum_{n=0}^{\infty} \frac{R^{2 n}}{(2 n+1) !} \frac{\partial^{n} u\left(\mathbf{x}_{0}, t\right)}{\partial t^{n}}
\end{aligned}
$$

for $R$ sufficiently small. We shall refer to (2) as the mean value theorem for the heat equation.

Now suppose $u$ achieves its maximum at a point $\left(\mathbf{x}_{0}, t_{0}\right)$ as stated in the theorem. Suppose $u$ is not identically equal to $M$ in the component of the intersection of $E$ with the plane $t=t_{0}$ that contains $\left(\mathbf{x}_{0}, t_{0}\right)$. Then there exists a sequence of positive numbers $\rho_{n}, \rho_{n} \rightarrow 0$, such that $S\left(\rho_{n}, t_{0}\right)<M$, since if not $u$ is identically equal to $M$ in a neighbourhood of $\left(\mathbf{x}_{0}, t_{0}\right)$ intersected with the plane $t=t_{0}$ and hence by analyticity $u$ is identically equal to $M$ in the entire component described above. We now note that for $R_{0}$ and $h$ sufficiently small, $S(R, t)$ is a spherically symmetric solution of the heat equation in the cylinder $R \leqq R_{0}, t_{0}-h \leqq t \leqq t_{0}$, and achieves its maximum value $M$ at $\left(0, t_{0}\right)$. Choose $R_{0}=\rho_{n_{0}}$ for $n_{0}$ sufficiently large. Then by the weak maximum principle for the heat equation (c.f. [1]) there exists a point $\left(R_{1}, t_{1}\right), 0 \leqq R_{1} \leqq \rho_{n_{0}}, t_{0}-h \leqq t_{1} \leqq t_{0}$, such that $S\left(R_{1}, t_{1}\right)=M$. Applying the above argument to the cylinder $R \leqq \rho_{n_{1}}, t_{1}+$ $\rho_{n_{1}} \leqq t \leqq t_{0}$, where $t_{1}+\rho_{n_{1}}<t_{0}, \rho_{n_{1}}<\rho_{n_{0}}, \quad$ yields a point $\left(R_{2}, t_{2}\right), \quad 0 \leqq R_{2} \leqq \rho_{n_{1}}$, $t_{1}+\rho_{n_{1}} \leqq t_{2}<t_{0}$, such that $S\left(R_{2}, t_{2}\right)=M$, and proceeding in this manner we have an infinite sequence of points $\left(R_{n}, t_{n}\right)$ such that $S\left(R_{n}, t_{n}\right)=M$ and whose only accumulation point is $\left(0, t_{0}\right)$. Since $\left(R_{n}, t_{n}\right)$ are maximum points, we have $S_{t}\left(R_{n}, t_{n}\right)=0$, and our choice of concentric cylinders implies that

$$
\left|\frac{R_{n+1}-R_{n}}{t_{n+1}-t_{n}}\right| \leqq 1
$$

for all $n$. From the mean value theorem for functions of several variables applied to $S_{t}(R, t)$ we now have that

$$
0=\frac{S_{R t}\left(R_{n}^{0}, t_{n}^{0}\right)\left(R_{n+1}-R_{n}\right)}{t_{n+1}-t_{n}}+S_{t t}\left(R_{n}^{0}, t_{n}^{0}\right)
$$

where $\left(R_{n}^{0}, t_{n}^{0}\right)$ is a point on the line segment joining $\left(R_{n}, t_{n}\right)$ to $\left(R_{n+1}, t_{n+1}\right)$, and since $S_{R t}\left(0, t_{0}\right)=0$ we have from (3) and (4) by letting $n$ tend to infinity that $S_{t t}\left(0, t_{0}\right)=$ $\left(\partial^{2} u\left(\mathbf{x}_{0}, t_{0}\right) / \partial t^{2}\right)=0$. Since $S\left(\rho_{n}, t_{0}\right)<M$ by hypothesis, $S\left(R, t_{n}\right)$ is not identically equal to $M$ for $R \leqq R_{0}$ and an appropriate infinite subsequence of the positive integers. Hence 
applying the above argument to $S(R, t)$ and the point $\left(R_{n}, t_{n}\right)$ instead of $u(x, t)$ and $\left(\mathrm{x}_{0}, t_{0}\right)$ we can conclude that $S_{t t}\left(R_{n}, t_{n}\right)=0$ for $n$ in this subsequence. Equation (4) is now valid for $S_{t}$ replaced by $S_{t t}$ and hence we can conclude that $S_{t t t}\left(0, t_{0}\right)=\left(\partial^{3} u\left(\mathbf{x}_{0}, t_{0}\right) / \partial t^{3}\right)$ $=0$. By induction we now have that all the derivatives of $u$ with respect to $t$ vanish at $\left(\mathrm{x}_{0}, t_{0}\right)$, and hence from the mean value theorem for the heat equation we have that $S\left(R, t_{0}\right)=M$ for all $R$ sufficiently small. But this contradicts the fact that $S\left(\rho_{n}, t_{0}\right)<M$ and hence we can conclude that $u$ is identically equal to $M$ in the component of the intersection of $E$ with the plane $t=t_{0}$ that contains $\left(\mathbf{x}_{0}, t_{0}\right)$.

We now complete the proof of the theorem by using a simple comparison argument due to Nirenberg (c.f. [2], p. 167). Let $\left(\mathrm{x}_{0}, t_{0}\right)$ be as above such that the vertical segment $\left(\mathbf{x}_{0}, t\right), t_{1} \leqq t \leqq t_{0}$ lies in $E$. Suppose there is a point $\left(\mathbf{x}_{0}, t_{2}\right), t_{1} \leqq t_{2}<t_{0}$, such that $u\left(\mathrm{x}_{0}, t_{2}\right)<M$ and let $\tau$ be the least upper bound of such values of $t_{2}$. Then by continuity $u\left(\mathbf{x}_{0}, \tau\right)=M$ and $u\left(\mathbf{x}_{0}, t\right)<M$ for $t_{2} \leqq t<\tau$. Then by the above analysis $u(\mathbf{x}, t)<M$ in some cylinder $\left|\mathbf{x}-\mathbf{x}_{0}\right| \leqq \varepsilon, t_{2} \leqq t<\tau$ and $u(\mathbf{x}, \tau)=M$ for $\left|\mathbf{x}-\mathbf{x}_{0}\right| \leqq \varepsilon$. We shall now obtain a contradiction. Let $D$ be a ball centred at $\left(\mathbf{x}_{0}, \tau\right)$ such that the lower half of $D$ is contained in the above cylinder and let $\Omega$ be that part of $D$ lying below the paraboloid $\left|\mathbf{x}-\mathbf{x}_{0}\right|^{2}+7(t-\tau)=0$. Then $\partial \Omega=C_{1}+C_{2}$ where $C_{1}$ is part of $\partial D$ and $C_{2}$ is part of the boundary of the paraboloid. Consider the function $v$ defined by

$$
v(\mathbf{x}, t)=u(\mathbf{x}, t)-\varepsilon\left(\left|\mathbf{x}-\mathbf{x}_{0}\right|^{2}+7(t-\tau)\right)
$$

where $\varepsilon>0$. Then $v_{t}-\Delta_{3} v=-\varepsilon<0$ and $v$ achieves its maximum in $\bar{\Omega}$. The point at which this maximum is achieved cannot be in $\Omega$ since at such a point $v_{t}=0$ and $\Delta_{3} v \leqq 0$. By choosing $\varepsilon$ sufficiently small we see that $v<M$ on $C_{1}, v \leqq M$ on $C_{2}$, and since $v=u$ on $C_{2}$ we can conclude that the maximum of $v$ is $M$ and occurs at $\left(\mathbf{x}_{0}, \tau\right)$. Hence at $\left(\mathbf{x}_{0}, \tau\right)$ we have $(\partial v / \partial t) \geqq 0$. But since $(\partial u / \partial t)\left(\mathbf{x}_{0}, \tau\right)=0$ we see from (5) that $(\partial v / \partial t)\left(\mathbf{x}_{0}, \tau\right)=$ $-7_{\varepsilon}<0$, and thus we obtain the desired contradiction. Hence we can conclude that $u=$ $M$ on every vertical line segment whose upper end point is an interior maximum point of $u$.

We note in closing that a strong minimum principle for the heat equation can be obtained by simply replacing $u$ by $-u$ in the above theorem.

\section{REFERENCES}

1. F. John, Partial Differential Equations, Third Edition (Springer-Verlag, Berlin, 1978).

2. M. Protter and H. Weingerger, Maximum Principles in Differential Equations (Prentice Hall, Englewood Cliffs, 1967).

Department of Mathematical Sciences

UNIVERSITY OF DELAWARE

Newark, Delaware 19716 